\title{
Effects of trimester-specific and total gestational weight gain on children's anthropometrics
}

\author{
Otmar Bayer ${ }^{1 *}$, Regina Ensenauer ${ }^{2,3}$, Ina Nehring ${ }^{1}$ and Rüdiger von Kries ${ }^{1}$
}

\begin{abstract}
Background: Gestational weight gain (GWG) has been shown to be a risk factor for overweight in offspring. Aim of this study was to quantify the contributions of trimester-specific and total GWG on offspring's BMI and waist circumference (WC). This is of interest for the design of interventions targeted at women showing a high GWG in early pregnancy.

Methods: In a retrospective cohort study data on GWG (total and by trimester, exposure), a number of potential confounders, and children's BMI z-scores and WC (outcomes) were analyzed using structural equation models to disentangle the trimester-specific direct effects of GWG and indirect effects mediated via total GWG.

Results: 7313 mother child pairs with a children's mean age of 5.81 years were analyzed. Total effects (indirect + direct) of GWG (kg/week) on children's BMI z-score and WC $(\mathrm{cm})$ were observed in all trimesters, most prominently in the second. The longitudinal effect of GWG is a composite of trimester-specific direct effects (on BMl: 0.105, $0.255,0.002$, on WC: $0.538,1.64,0.308$ ) and total GWG (on BMI 0.608, on WC: 1.03) at the end of pregnancy.

Conclusions: Both trimester-specific priming and total GWG explained offspring's anthropometrics. The results indicate, that reversal from additional weight gain attained early in pregnancy resulting in normal total GWG at the end of pregnancy might still contribute to a substantial reduction of offspring's BMI and WC.
\end{abstract}

Keywords: Gestational weight gain, Pregnancy, Priming, Children, Overweight, Obesity, Structural equation models

\section{Background}

Weight gain in pregnancy is associated with different perinatal [1] and longterm outcomes in mothers and offspring [2-4], which is underlined by recommendations for gestational weight gain (GWG) by the Institute of Medicine [5]. Several studies observed associations between GWG and children's anthropometric outcomes [6-10]. More recently, studies revisited this association subdividing the pregnancy period, raising the question, whether there are critical periods for GWG with respect to priming children's BMI [11]. As there is a strong need for early prevention concepts in counteracting childhood overweight and obesity, it is of major interest to define the point in time when an intervention against excessive GWG should be delivered. Recommending strategies to reduce GWG for all women irrespective of their individual risk (scattergun

\footnotetext{
* Correspondence: Otmar.Bayer@Irz.uni-muenchen.de

${ }^{1}$ Institute for Social Paediatrics and Adolescent Medicine,

Ludwig-Maximilians-Universität München, Haydnstr. 5/4. OG, 80336 Munich, Germany

Full list of author information is available at the end of the article
}

approach) has the advantage of the earliest intervention possible but is associated with the risk of increasing inadequate GWG [12]. Targeting interventions would require to first observe the pregnant women's weight gain in order to decide, whether they excessively gain weight or not. As a consequence, interventions would be delayed, leading to a dilemma, if later GWG would not affect the desired outcome, in this case offspring's BMI.

We therefore revisited the research question, if there are critical periods during pregnancy with respect to GWG and programming of a risk of overweight and abdominal adiposity in the offspring. Further we aimed to elucidate to what extent these are direct effects of GWG in the respective periods, that cannot be explained by their contribution to total weight gain.

\section{Methods \\ Participants and data sources}

From October 2009 to June 2011, all children attending the mandatory school entry health examinations in 6 regions in

\section{Biomed Central}


Bavaria, both urban and rural, were invited to participate in this retrospective cohort study (PEPO: PErinatal Prevention of Obesity). Detailed information about sample recruitment and data collection has been published previously [13].

Prior to the school entry health examinations, information leaflets were sent to all families. Mothers and their children were invited to take part in the study, and a questionnaire on sociodemographic and other risk factors for childhood overweight was completed at the time of examination. Anthropometric data were collected by trained study nurses on the day of the school entry health examination. The child's weight was measured, wearing underwear, with a calibrated electronic scale (Seca, Birmingham, UK) with an accuracy of $0.1 \mathrm{~kg}$. Height was measured, without wearing shoes, with an accuracy of $0.1 \mathrm{~cm}$ using a stadiometer (Seca, Birmingham, UK). Waist cirumference was measured to the nearest $0.1 \mathrm{~cm}$ midway between the lowest rib and the top of the iliac crest according to the WHO recommendations [14]. Measurements were carried out at the end of a gentle expiration after an inelastic tape (Seca) was placed directly on the skin of the child who was standing balanced on both feet, without wearing shoes, and whose arms were hanging freely. Measurements were carried out three times and the mean was used for analysis.

Data on maternal pre-pregnancy weight and height and GWG in the first, second and third trimesters, diabetes and gestational diabetes were obtained from the "maternity pass." In Germany, a "maternity pass" is issued to every pregnant woman at her first antenatal visit to the gynaecologist. The mothers keep the "maternity pass" as a personal document for the first and all subsequent pregnancies. The document contains health care information relevant to the pregnancy, and also includes data on serial weight measurements over the course of pregnancy documented by the consulted physician. On the day of the school entry health examination, trained study nurses copied weight-related data from the "maternity pass".

The study protocol was approved by the ethics committee of the Ludwig-Maximilians Universität München, and signed informed consent was obtained from all participants.

\section{Explanatory variables}

The exposures of interest were trimester-specific and total GWG. First trimester (t1) weight gain was calculated by subtracting pre-pregnancy weight from the weight measured between the first and 13th week. Second trimester (t2) weight gain was calculated by subtracting the weight measured in $\mathrm{t} 1$ from the weight measured between week 14 and 26. Third-trimester (t3) weight gain was calculated by subtracting the weight measured in $\mathrm{t} 2$ from the weight measured between week 27 and 40. Measurements outside these time frames were excluded.

The following potential confounding factors, which were ascertained by use of a questionnaire including questions from the KiGGS study [15] and a recent Bavarian study on breastfeeding [16] were used in the analysis [17]: Breastfeeding was dichotomized as "at least one month full-time without interruption" and "less than one month full-time". Maternal smoking was dichotomized as "at no time during pregnancy" or "any time during pregnancy". Parental SES was defined in three categories using an additive index based on maternal and paternal educational background and current type of maternal and paternal employment [18]. Maternal age and pre-pregnancy BMI were treated as continuous variables.

\section{Statistical analysis}

To disentangle the effects of trimester-specific and total GWG (which are highly correlated due to the contribution of each trimesters' weight gain to total GWG) on the offspring's BMI and waist circumference in childhood, we set up a structural equation model depicted in Figure 1. Structural equation models deal with highly correlated measurements, and make it possible to obtain estimates for direct, indirect, and total effects. The indirect effect is the part of the effect mediated through other covariates on the path (e. g. GWG at t1 contributing to

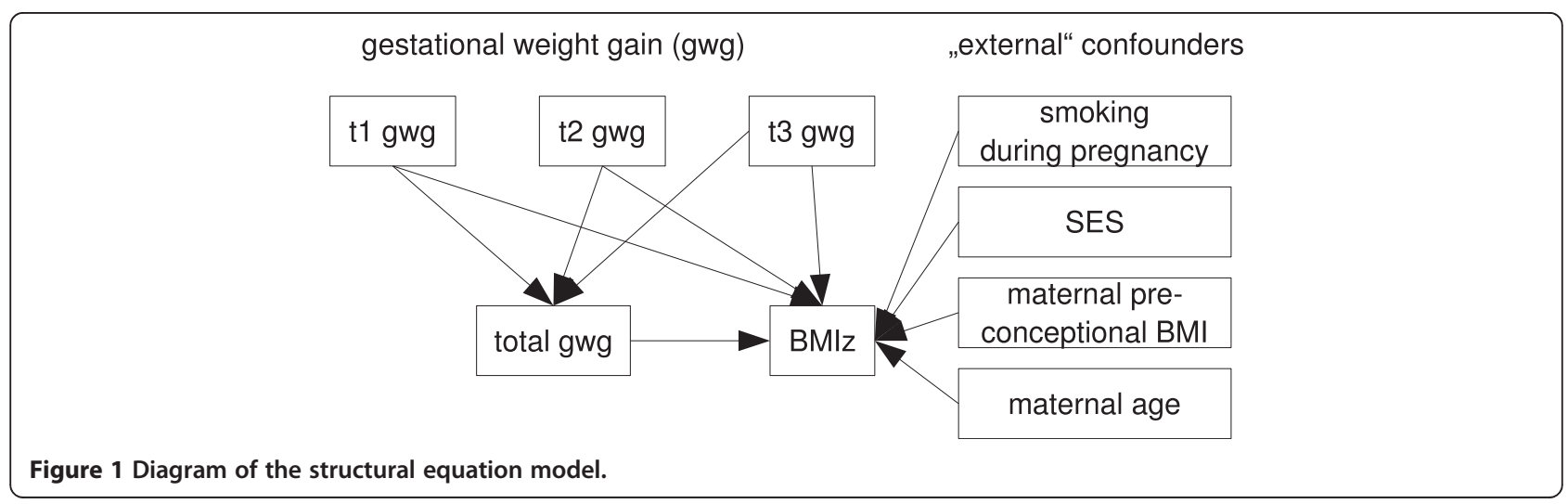


total GWG, which in turn affects offspring's BMI). The total effect is the sum of direct and indirect effect. To adjust for potential confounding by parental SES, maternal smoking during pregnancy, age, pre-pregnancy BMI, these variables were included to directly affect offspring's BMI z-score (Figure 1). Since there were no applicable reference values to convert the waist circumference data to $\mathrm{z}$-scores, the untransformed values in $\mathrm{cm}$ were used, additionally adjusting for children's age and sex in the model. In the BMI model the use of age and sex specific BMI z-scores inherently corrects for these two variables.

Standard errors of indirect effects were estimated using Sobel's (1982) method [19].

\section{Results}

The numbers of recruited and included subjects are illustrated in Figure 2. The median times of GWG measurements included were 7, 24, and 36 weeks after conception for $\mathrm{t} 1$, $\mathrm{t} 2$, and $\mathrm{t} 3$ (Figure 3 ).

Data on BMI z-score, WC and maternal GWG were available for 7769 children, additional information on external confounders (shown in Figure 1) for 7313, which served as the dataset for analysis. At follow-up the children were $5.81 \pm 0.37$ (mean \pm std) years of age, 3580 (49.0\%) were girls. Further sample description can be found in Table 1. Children excluded had a 0.06 higher BMI z-score, and a $0.38 \mathrm{~cm}$ higher waist circumference, which are small but statistically significant mean differences. Among the excluded participants the proportion with high parental SES and mothers who smoked during pregnancy was higher (see Table 1). The results from structural equation modeling are presented in Table 2 and can be interpreted as BMI z-score (standard deviations) per GWG (kg/week). For example, one kg more maternal weight gain during t 1 results on average in a 0.2 higher

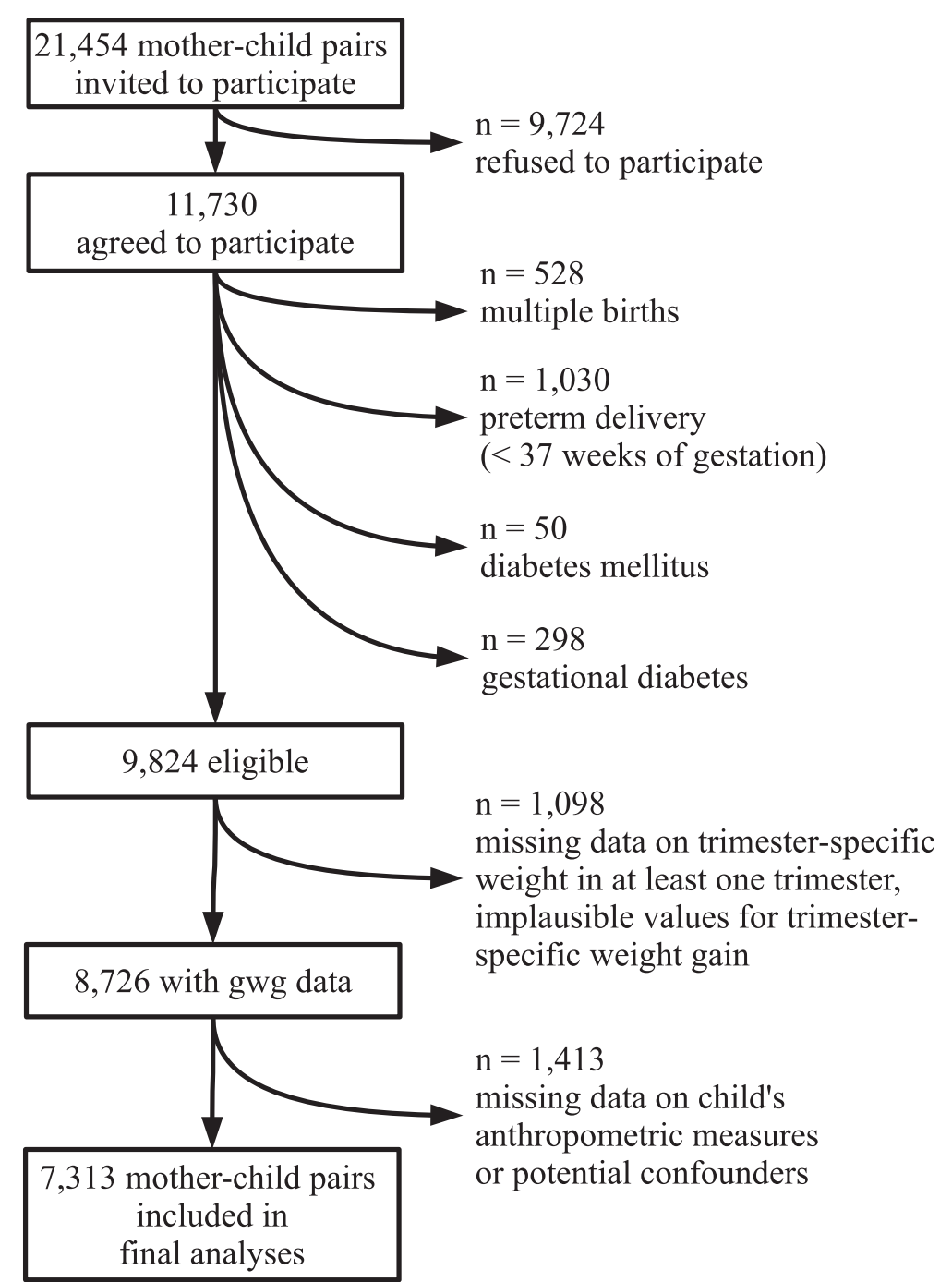

Figure 2 Participants flow diagram. 


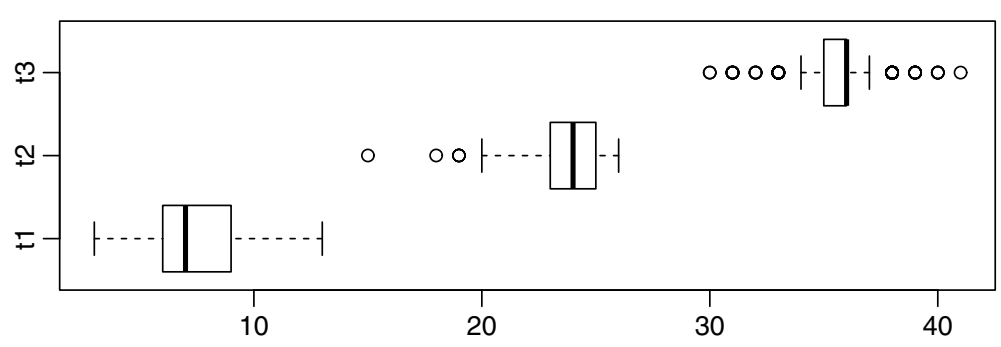

Figure 3 Time (in weeks) of weight measurement during pregnancy. Shown are the median ( $\mathrm{t} 1: 7, \mathrm{t} 2: 24, \mathrm{t} 3: 36)$ surrounded by the interquartile range (box), the most extreme values within 1.5 interquartile ranges from the box (whiskers) and outliers (circles).

BMI z-score in the child. This total effect is equally contributed by the direct and indirect effect. In summary, there is an effect of GWG on children's BMI z-score in all trimesters, though most prominent in $\mathrm{t} 2$. While the effect is half a direct, and half an indirect effect in $t 1$ and $t 2$, for t3 it is a completely indirect effect, mediated by total GWG.

For waist circumference, the same pattern for the total effects was observed. However, the contribution of the direct effects appeared larger in all three trimesters, while the indirect effects could not be detected as statistically significant (alpha $=0.05)$.

\section{Discussion}

Structural equation modeling of 7313 mother child pairs suggests that weight gained at any time during pregnancy directly or indirectly (mediated via total GWG) affects offspring's anthropometrics.

A substantial proportion of the effect of the $1^{\text {st }}$ and $2^{\text {nd }}$ trimester GWG on childhood BMI is mediated by total GWG. Therefore, identification of excessive GWG early in pregnancy might not justify complacency: achieving adequate total GWG will still result in lower offspring BMI and waist circumference.

The present analysis differs in two ways from previous studies published by our group $[17,20]$. 1) Here, we used continuous instead of dichotomized explanatory $(\mathrm{kg} /$ week instead of excessive weight gain) and outcome (BMI z-score and waist circumference instead of overweight) variables. 2) Previous analyses compared cumulative GWG to the respective trimester-specific cutoffs, thus carrying information from previous trimesters (e. g. whether GWG exceeds the cutoff in $\mathrm{t} 3$ also depends on

Table 1 Sample description

\begin{tabular}{|c|c|c|c|}
\hline Variable & $\begin{array}{l}\text { Mean } \pm \text { std or } n \text { (percentage) } \\
\text { eligible } n=9824\end{array}$ & $\begin{array}{l}\text { Mean } \pm \text { std or } n \text { (percentage) } \\
\text { included in model } n=7313\end{array}$ & $\begin{array}{l}\text { Mean } \pm \text { std or } n \text { (percentage) excluded } \\
\text { from model } n=2511\end{array}$ \\
\hline BMI z-score at 6 y & $0.02 \pm 1.04$ & $0 \pm 1.03$ & $0.07 \pm 1.1$ \\
\hline $\begin{array}{l}\text { Waist circumference in } \mathrm{cm} \\
\text { at } 6 \mathrm{y}\end{array}$ & $52.6 \pm 4.5$ & $52.5 \pm 4.3$ & $52.8 \pm 4.9$ \\
\hline Weekly GWG at t1 in kg & $0.1 \pm 0.3$ & $0.2 \pm 0.3$ & $0.1 \pm 0.4$ \\
\hline Weekly GWG at t2 in kg & $0.4 \pm 0.2$ & $0.4 \pm 0.2$ & $0.4 \pm 0.2$ \\
\hline Weekly GWG at t3 in kg & $0.5 \pm 0.2$ & $0.5 \pm 0.2$ & $0.5 \pm 0.2$ \\
\hline Maternal age in years & $28.9 \pm 5.3$ & $29 \pm 5.1$ & $28.7 \pm 5.7$ \\
\hline $\begin{array}{l}\text { Maternal pre-pregnancy } \\
\text { BMI in } \mathrm{kg} / \mathrm{m}^{2}\end{array}$ & $23.4 \pm 4.2$ & $23.4 \pm 4.2$ & $23.4 \pm 4.4$ \\
\hline $\begin{array}{l}\text { Underweight mothers (BMI } \\
<18.5 \mathrm{~kg} / \mathrm{m}^{2} \text { ) }\end{array}$ & $465(5.1)$ & $343(4.7)$ & $122(6.5)$ \\
\hline $\begin{array}{l}\text { Overweight mothers (BMl } \\
>25 \mathrm{~kg} / \mathrm{m}^{2} \text { ) }\end{array}$ & $2377(25.8)$ & $1869(25.6)$ & $508(27)$ \\
\hline $\begin{array}{l}\text { Obese mothers (BMI > } \\
\left.25 \mathrm{~kg} / \mathrm{m}^{2}\right)\end{array}$ & $716(7.8)$ & $555(7.6)$ & $161(8.6)$ \\
\hline $\begin{array}{l}\text { Breastfeeding, at least one } \\
\text { month fulltime }\end{array}$ & $6703(73.2)$ & $5043(73.2)$ & $1660(73)$ \\
\hline $\begin{array}{l}\text { Smoking, any time during } \\
\text { pregnancy }\end{array}$ & $1162(12)$ & $806(11)$ & $356(15.2)$ \\
\hline $\begin{array}{l}\text { Parental SES "high"/ } \\
\text { "medium"/"low" }\end{array}$ & $\begin{array}{l}3129(33.2) / 3688(39.1) / 2614 \\
(27.7)\end{array}$ & $2313(31.6) / 2933(40.1) / 2067$ (28.3) & $816(38.5) / 755$ (35.6)/547 (25.8) \\
\hline
\end{tabular}




$\begin{aligned} & \text { Table } 2 \text { Effects obtained from structural equation } \\
& \text { modeling }\end{aligned}$
\begin{tabular}{llll}
\hline Effects on BMI z-scores (i. e. std) per GWG (kg/week) \\
\hline Period & $\begin{array}{l}\text { Direct } \\
\text { effect } \pm \\
\text { stderr }\end{array}$ & $\begin{array}{l}\text { Indirect } \\
\text { effect } \pm \\
\text { stderr }\end{array}$ & $\begin{array}{l}\text { Total effect } \pm \text { stderr } \\
\text { (estimated via OLS } \\
\text { regression) }\end{array}$ \\
\hline t1 & $0.105 \pm 0.060^{*}$ & $0.104 \pm 0.043^{*}$ & $0.208 \pm 0.042^{*}$ \\
t2 & $0.255 \pm 0.116^{*}$ & $0.241 \pm 0.100^{*}$ & $0.498 \pm 0.064^{*}$ \\
t3 & $0.002 \pm 0.098$ & $0.199 \pm 0.083^{*}$ & $0.198 \pm 0.056^{*}$ \\
Total gwg & $0.608 \pm 0.252^{*}$ & & \\
\hline Effects on waist circumference (cm) per GWG & (kg/week) \\
\hline Period & $\begin{array}{l}\text { Direct } \\
\text { effect } \pm \\
\text { stderr }\end{array}$ & $\begin{array}{l}\text { Indirect } \\
\text { effect } \pm \\
\text { stderr }\end{array}$ & $\begin{array}{l}\text { Total effect } \pm \text { stderr } \\
\text { (estimated via OLS } \\
\text { regression) }\end{array}$ \\
\hline t1 & $0.538 \pm 0.254^{*}$ & $0.176 \pm 0.181$ & $0.714 \pm 0.179^{*}$ \\
t2 & $1.644 \pm 0.489^{*}$ & $0.408 \pm 0.421$ & $2.055 \pm 0.268^{*}$ \\
t3 & $0.308 \pm 0.413$ & $0.337 \pm 0.347$ & $0.641 \pm 0.237^{*}$ \\
Total gwg & $1.03 \pm 1.062$ & & \\
\hline
\end{tabular}

The rows in this table represent the trimester-specific effects of GWG broken down to the direct part and the part mediated via total GWG. Significant ( $p<$ 0.05 ) are marked by *. The first column can be interpreted longitudinally over the whole pregnancy period: a mother gaining additional weight of $1 \mathrm{~kg} /$ week in $\mathrm{t} 1$ and $\mathrm{t} 2$ and compensating this gain in $\mathrm{t} 3$ arriving at no additional total GWG attains the direct effects of $\mathrm{t} 1$ and $\mathrm{t} 2(0.105+0.255=0.36)$ accounting for an increment in offspring's BMI of $0.36 \mathrm{z}$-scores, but avoids the effect of total GWG. A mother continuing to gain additional weight of $1 \mathrm{~kg} /$ week in $\mathrm{t} 3$ would attain the direct effects of $\mathrm{t} 1-\mathrm{t} 3$ plus the effect of total GWG $(0.105+0.255+0+0.608=0.97)$.

GWG in $\mathrm{t} 1$ and $\mathrm{t} 2$ ). The structural equation modeling allows to disentangle trimester specific (direct) effects from the effect of total GWG.

In many mothers excessive GWG early in pregnancy will result in high total GWG. Indeed, as recently demonstrated $80 \%$ of the mothers with excessive GWG early in pregnancy show excessive GWG at the end of pregnancy [13]. Therefore, early GWG allows to identify women at high risk for excessive total GWG. This is important with respect to the design of preventive programs against high GWG.

Such intervention programs might be delivered to all pregnant women as close to conception as possible. As shown in recent meta analyses $[1,21]$ such interventions are efficacious in reducing GWG. Such a shift in GWG of the total population, however, will also increase the proportion of pregnancies with inadequate (i. e. too low) GWG, which has been shown to be associated with unintended outcomes $[1,12]$. Therefore, targeted interventions at women with high early GWG would be tempting, if still efficacious with respect to reducing overweight and abdominal adiposity in the offspring.

Our data confirm a strong effect of GWG in mid pregnancy as reported by others [11]. In contrast to the findings of Anderson et al. we found a still significant effect of t3 GWG on offspring's BMI and waist circumference.
This discrepancy might reflect different time periods considered: in Anderson's study t2 extends to week 30 (max. week 32 ) with $\mathrm{t} 3$ consequently capturing the last 10 (8) weeks of pregnancy only. In our study the t3 period was in median between the $24^{\text {th }}$ and $36^{\text {th }}$ week of pregnancy. Another important difference is given by the analysis, as in Anderson's structural equation model the indirect effect via total GWG was not considered. This, however, is essential to disentangle potential direct and indirect effects of GWG in pregnancy: if the entire effect on offspring's BMI were mediated by direct effects via priming early in pregnancy, efforts to normalize total GWG later in pregnancy would be ineffective with respect to children's BMI. We could not identify any further study addressing the association between trimesterspecific GWG on offspring's BMI or overweight.

Since BMI reflects both fat and lean mass, we additionally considered children's waist circumference as a measure of abdominal adiposity. A high waist circumference has been shown to be related to cardiovascular risk factors such as total cholesterol and blood pressure in school children [22]. We therefore repeated our analysis using waist circumference instead of BMI as outcome variable. This revealed the same pattern of total effects, although direct effects were more prominent than in the BMI analysis. Nevertheless, the conclusion for interventions remain the same: A woman gaining excess weight in the first half of pregnancy and compensating this excess gain in the second half arriving at a normal GWG contributes markedly to a lower offspring waist circumference compared to a women continuing to gain excess weight throughout pregnancy (this can be calculated similar to the example given in Table 2).

We adjusted the models for a number of confounding variables, however, such adjustment is incomplete. There were no anthropometric measurements of the father available, which is a limitation. We sought to include breastfeeding (see Explanatory variables), however this would have led to exclusion of further 432 children. Therefore, we did not include breastfeeding in our main models but conducted a sensitivity analysis including this variable, which did not substantially change the results: for $\mathrm{t} 3$ the direct/indirect effects \pm standard errors were $0.009 \pm 0.101 / 0.173 \pm 0.085$ on BMI $z$-score, and $0.310 \pm 0.421 / 0.290 \pm 0.354$ on waist circumference. The effect of total GWG was $0.531 \pm 0.261$ on BMI z-score, and $0.893 \pm 1.090$ on waist circumference.

A strength of our study is the ascertainment of maternal weight - the primary exposure variable - from medical records on measured weight. An exception to this is the pre-pregnancy weight, which was recalled at the first antenatal visit. The imprecision introduced by this limitation could introduce a bias towards null and might contribute to the weak effect of $\mathrm{t} 1$ GWG. However, the self- 
reported pre-pregnancy weight and the first measured weight in pregnancy correlated very well $(\mathrm{r}=0.988)$.

The availability of two relevant outcome variables BMI and waist circumference is a further strength. The data to compute the outcome variables were measured in a standardized way at the school entry health examination.

Our findings underline the importance of the established risk factor total GWG. Although trimester-specific weight gains allow for more precise prediction of offspring BMI, total GWG is a simpler and still reasonable predictor, as there is a considerable time-independent effect.

\section{Conclusion}

The effect of GWG on offspring's BMI is not confined to the first two trimesters. Effects attributable to early GWG are partially mediated by total GWG and thus may be reversed if excessive total GWG is avoided. Therefore, interventions targeted at women with excessive GWG identified in early pregnancy appear warranted.

\section{Abbreviations \\ GWG: Gestational weight gain; BMI: Body mass index; WC: Waist circumference; WHO: World Health Organisation; KiGGS: Kinder- und Jugendgesundheitssurvey (The German Health Survey for Children and Adolescents); SES: Socio-economic status; std: Standard deviation; stderr: Standard error.}

\section{Competing interests}

The authors declare that they have no competing interests.

\section{Authors' contributions}

The authors' responsibilities were as follows: RvK and OB designed the research, RE designed and conducted the PEPO study, and was responsible for the data acquisition, OB performed statistical analysis, OB, IN, RE and RvK wrote the manuscript. All authors read and approved the final manuscript.

\section{Acknowledgements}

We thank the local health authorities, study nurses and the families for their participation and support.

We thank Andrea Chmitorz for preparatory work on the data set. The study was part of the PEPO consortium of the Competence Network Obesity supported by the German Federal Ministry of Education and Research (BMBF). Funding sources to RE were German Federal Ministry of Education and Research (BMBF) Grant 0315088 and Grant 01 Gl0820 (project coordination and data analysis), and Bavarian State Ministry of the Environment and Public Health Grant LP00124 (data acquisition at school entry health examination). OB was supported by LMUinnovativ research priority project MCHealth (sub-project II).

\section{Author details \\ ${ }^{1}$ Institute for Social Paediatrics and Adolescent Medicine, Ludwig-Maximilians-Universität München, Haydnstr. 5/4. OG, 80336 Munich, Germany. ${ }^{2}$ Research Center, Dr. von Hauner Children's Hospital, Ludwig-Maximilians-Universität München, Munich, Germany. ${ }^{3}$ Experimental Pediatrics, Department of General Pediatrics, Neonatology and Pediatric Cardiology, University Children's Hospital, Heinrich-Heine-Universität Düsseldorf, Düsseldorf, Germany.}

Received: 29 April 2013 Accepted: 4 June 2014 Published: 8 October 2014

\section{References}

1. Thangaratinam S, Rogozinska E, Jolly K, Glinkowski S, Roseboom T, Tomlinson JW, Kunz R, Mol BW, Coomarasamy A, Khan KS: Effects of interventions in pregnancy on maternal weight and obstetric outcomes: meta-analysis of randomised evidence. BMJ 2012, 344:e2088.

2. Keppel KG, Taffel SM: Pregnancy-related weight gain and retention: implications of the 1990 Institute of Medicine guidelines. Am J Public Health 1993, 83:1100-1103.

3. Fraser A, Tilling K, Macdonald-Wallis C, Sattar N, Brion M-J, Benfield L, Ness A, Deanfield J, Hingorani A, Nelson SM, Smith GD, Lawlor DA: Association of maternal weight gain in pregnancy with offspring obesity and metabolic and vascular traits in childhood. Circulation 2010, 121:2557-2564.

4. Nehring I, Schmoll S, Beyerlein A, Hauner H, von Kries R: Gestational weight gain and long-term postpartum weight retention: a meta-analysis. Am J Clin Nutr 2011, 94:1225-1231.

5. Rasmussen K: Weight Gain During Pregnancy: Reexamining the Guidelines. Washington DC: Institute of Medicine; 2009.

6. Mamun AA, O'Callaghan M, Callaway L, Williams G, Najman J, Lawlor DA Associations of gestational weight gain with offspring body mass index and blood pressure at 21 years of age: evidence from a birth cohort study. Circulation 2009, 119:1720-1727.

7. Hinkle SN, Sharma AJ, Swan DW, Schieve LA, Ramakrishnan U, Stein AD: Excess gestational weight gain is associated with child adiposity among mothers with normal and overweight prepregnancy weight status. J Nutr 2012, 142:1851-1858.

8. Nehring I, Lehmann S, von Kries R: Gestational weight gain in accordance to the IOM/NRC criteria and the risk for childhood overweight: a metaanalysis. Pediatr Obes 2013, 8:218-224

9. Durmuş B, Arends LR, Ay L, Hokken-Koelega AC, Raat H, Hofman A, Steegers EAP, Jaddoe WW: Parental anthropometrics, early growth and the risk of overweight in pre-school children: the Generation R Study. Pediatr Obes 2012, 8:339-350

10. Dello Russo M, Ahrens W, De Vriendt T, Marild S, Molnar D, Moreno LA, Reeske A, Veidebaum T, Kourides YA, Barba G, Siani A: Gestational weight gain and adiposity, fat distribution, metabolic profile, and blood pressure in offspring: the IDEFICS project. Int J Obes (Lond) 2013, 37:914-919.

11. Andersen CS, Gamborg M, Sørensen TIA, Nohr EA: Weight gain in different periods of pregnancy and offspring's body mass index at 7 years of age. Int J Pediatr Obes 2011, 6:e179-e186.

12. Von Kries $R$, Nehring I: Reproductive endocrinology: reducing gestational weight gain-a panacea? Nat Rev Endocrinol 2012, 8:452-454.

13. Chmitorz A, von Kries R, Rasmussen KM, Nehring I, Ensenauer R: Do trimester-specific cutoffs predict whether women ultimately stay within the Institute of Medicine/National Research Council guidelines for gestational weight gain? Findings of a retrospective cohort study. Am J Clin Nutr 2012, 95:1432-1437.

14. World Health Organisation: WHO STEPS Surveillance. Guide to Physical Measurements (Step 2). Geneva: WHO; 2008.

15. Von Kries R, Ensenauer R, Beyerlein A, Amann-Gassner U, Hauner $H_{\text {, }}$ Rosario AS: Gestational weight gain and overweight in children: results from the cross-sectional German KiGGS study. Int J Pediatr Obes 2011, 6:45-54.

16. Kohlhuber M, Rebhan B, Schwegler U, Koletzko B, Fromme H: Breastfeeding rates and duration in Germany: a Bavarian cohort study. Br J Nutr 2008, 99:1127-1132

17. Ensenauer R, Chmitorz A, Riedel C, Fenske N, Hauner H, Nennstiel Ratzel U, von Kries R: Effects of suboptimal or excessive gestational weight gain on childhood overweight and abdominal adiposity: results from a retrospective cohort study. Int J Obes (Lond) 2013 37:505-512.

18. Böhm A, Ellsässer G, Lüdecke K: The Brandenburg social index: a tool for health and social reporting at regional and communal levels in the analysis of data of school beginners. Gesundheitswesen 2007, 69:555-559.

19. Preacher KJ, Hayes AF: SPSS and SAS procedures for estimating indirect effects in simple mediation models. Behavior Research Methods 2004, 36:717-731.

20. Von Kries R, Chmitorz A, Rasmussen KM, Bayer O, Ensenauer R: Late pregnancy reversal from excessive gestational weight gain lowers risk of childhood overweight - a cohort study. Obesity (Silver Spring) 2013 21:1232-1237. 
21. Streuling I, Beyerlein A, von Kries R: Can gestational weight gain be modified by increasing physical activity and diet counseling? A meta-analysis of interventional trials. Am J Clin Nutr 2010, 92:678-687.

22. Bekkers MBM, Brunekreef B, Koppelman GH, Kerkhof M, de Jongste JC, Smit $\mathrm{HA}$, Wijga AH: BMI and waist circumference; cross-sectional and prospective associations with blood pressure and cholesterol in 12-year-olds. PLOS ONE 2012, 7:e51801.

doi:10.1186/1471-2393-14-351

Cite this article as: Bayer et al.: Effects of trimester-specific and total gestational weight gain on children's anthropometrics. BMC Pregnancy and Childbirth 2014 14:351.

\section{Submit your next manuscript to BioMed Central and take full advantage of:}

- Convenient online submission

- Thorough peer review

- No space constraints or color figure charges

- Immediate publication on acceptance

- Inclusion in PubMed, CAS, Scopus and Google Scholar

- Research which is freely available for redistribution 